Editorial

\title{
La Psicología del Tránsito, una necesidad urgente en México: breve revisión del panora- ma nacional sobre tasas de mortalidad asociada a los accidentes de tránsito
}

\section{The Psychology of Transit, an urgent need in Mexico: A brief revision of the national panorama on mortality rates associated with transit accidents}

\author{
Carro Pérez, Ennio Héctor ${ }^{1}$
}

De acuerdo con el Decenio de Acción para la Seguridad Vial 2011-2020 emitido por la Organización Mundial de la Salud (OMS, 2011), nos encontramos a escasos dos años de que el plazo establecido en este se cumpla, por lo que la propuesta del gobierno mexicano de reducir en un 50\% las muertes por accidentes de tránsito (Estrategia Nacional de Seguridad Vial 2011-2020, 2011; Declaración de México para la Seguridad Vial: la ruta para salvar vidas en Iberoamérica y el Caribe, 2011), se vislumbra difícil de ser alcanzada. De acuerdo con el Secretariado Técnico del Consejo Nacional para la Prevención de Accidentes, de la Secretaría de Salud (STCONAPRA) (2018) la tasa de mortalidad por accidentes de tránsito no ha variado más allá de una décima desde el 2014, año en que la mortalidad fue de 13.3 fallecimientos por cada 100000 habitantes, la cual se repitió en 2015, y en 2016 fue de 13.2 por cada 100000 habitantes, lo que implica una desaceleración de la mortalidad respecto al periodo comprendido entre 2009 y 2013, donde la reducción en la tasa de mortalidad fue de 2.4 fallecimientos (STCONAPRA, 2013, 2016) a pesar del repunte en dos décimas ocurrido en el año 2012 (Ver Tabla 1). De tal manera, en términos porcentuales la reducción en la tasa de mortalidad de 2009 a 2016 es de $16.45 \%$, respecto a los falleci-

\footnotetext{
${ }^{1}$ Universidad Autónoma de Tamaulipas. Facultad de Derecho y Ciencias Sociales, Centro de Investigación y Desarrollo Tecnológico Aplicado al Comportamiento, Tampico, Tamaulipas, México.

*Correspondencia: ecarro@docentes.uat.edu.mx
} 
Carro, E.

mientos netos la reducción porcentual fue de $9.17 \%$, a pesar de ello, de acuerdo a las estimaciones del STCONAPRA (2018), la cifra neta de muertes en 2016 supera en 3,370 decesos a la meta fijada para el mismo año de 12,815 muertes.

De igual manera, en la información anterior, se puede advertir que los fallecimientos netos han tenido un comportamiento distinto respecto a la tasa de mortalidad, sí bien esta última parece haberse estabilizado a partir del año 2013, las muertes por accidentes de tránsito han tenido variaciones más sobresalientes, así en los años 2013 y 2014 la cantidad de ellos es muy similar, pero en 2015 y 2016 van gradualmente en aumento. Al calcular la proporción que ocupan los fallecimientos respecto a los accidentes, pode-

Tabla 1.

Números nacionales de accidentalidad, heridos, muertes y tasa de mortalidad por accidentes de tránsito 2009-2016*

\begin{tabular}{lcccccccc}
\hline & 2009 & 2010 & 2011 & 2012 & $2013^{* *}$ & 2014 & 2015 & 2016 \\
\hline Accidentes & 458,06 & 455,628 & 412,087 & 414,62 & 407,808 & 396,25 & 399,330 & 372,61 \\
& 3 & & & 7 & & 4 & 8 & 8 \\
Heridos & 185,54 & 172,650 & 161,780 & 153,68 & 143,829 & 134,81 & 122,940 & 108,78 \\
& 9 & & & 5 & & 5 & & 9 \\
$\begin{array}{l}\text { Fallecimien- } \\
\text { tos }\end{array}$ & 17,820 & 16,559 & 16,615 & 17,102 & 15,853 & 15,886 & 16,039 & 16,185 \\
$\begin{array}{l}\text { Tasa de } \\
\text { mortalidad }\end{array}$ & 15.8 & 14.5 & 14.4 & 14.6 & 13.4 & 13.3 & 13.3 & 13.2 \\
\hline
\end{tabular}

* Desarrollada a partir de los datos de STCONAPRA/Secretaría de Salud (2013, 2016, 2018).

**Las cifras para este año son discrepantes en los informes de seguridad vial observados para los años 2013, 2015 y 2017.

Se ha preferido utilizar las cifras del informe 2017 (STCONAPRA, 2018).

mos observar que en el período del 2012 al 2016, salvo el año 2013, la proporción de fallecidos por cada cien accidentes es mayor que en los años anteriores a la puesta en mar- cha de la Estrategia Nacional de Seguridad Vial 2011-2020 (2011). (Ver Tabla 2). De tal manera, si bien los accidentes han sufrido una reducción del $18.65 \%$ aproximadamente des-

Tabla 2.

Muertes por cada cien accidentes de tránsito en México, 2009-2016*

\begin{tabular}{lcccccccc}
\hline & 2009 & 2010 & 2011 & 2012 & 2013 & 2014 & 2015 & 2016 \\
\hline $\begin{array}{l}\text { Muertes por cada } \\
\text { cien accidentes }\end{array}$ & 3.89 & 3.63 & 4.03 & 4.12 & 3.88 & 4.00 & 4.01 & 4.34 \\
\hline
\end{tabular}


de 2009, han producido más muertes que antes de 2011, pareciera que la letalidad de los mismos se ha incrementado con el tiempo.

Realizando una análisis más detallado de las cifras observadas sobre defunciones y las metas sobre las mismas a partir del 2011, año en que se inició la Estrategia Nacional de
Seguridad Vial 2011-2020, se puede observar que se requería un descenso anual porcentual entre el 4 y el 5 por ciento, sin embargo, de un año a otro se ha verificado un incremento porcentual mínimo, a excepción de la reducción en un 7.3\% ocurrida en el 2013 respecto al año 2012. (Ver Tabla 3).

Tabla 3.

Muertes meta y observadas por accidentes de tránsito y porcentaje de variación interanual, 2011-2016*

\begin{tabular}{lrrrrrr}
\hline & $2011 * *$ & 2012 & 2013 & $2014 * * *$ & 2015 & 2016 \\
\hline Meta & 16,615 & 15,855 & 15,095 & 14,335 & 13,575 & 12,815 \\
\% var. interanual & & $-4.57 * * * *$ & -4.79 & -5.03 & -5.30 & -5.60 \\
\cline { 2 - 7 } Observados & 16,615 & 17,102 & 15,853 & 15,886 & 16,039 & 16,185 \\
\% var. interanual & & 2.93 & -7.30 & 0.20 & 0.96 & 0.91 \\
\hline
\end{tabular}

* Desarrollada a partir de los datos de STCONAPRA/Secretaría de Salud (2018).

** Las cifras para este año, entre meta y observados, coinciden porque es el punto de partida para la proyección de las futuras reducciones en la cantidad de defunciones.

***Las cifras para este año son discrepantes en el informe de seguridad vial para el año 2017 (STCONAPRA, 2018). Se ha preferido utilizar la cifra más constante dentro del informe.

****Los valores negativos del porcentaje de variación interanual, representan una reducción de un año a otro, mientras que los positivos un incremento.

Sí bien los datos nacionales no muestran un gran avance en la reducción de las muertes por accidentes de tránsito, en el mejor de los casos describen un panorama estabilizado, los datos entre las entidades federativas son menos homogéneos. Así, las tasas de mortalidad por entidad federativa ofrecidas por el mismo STCONAPRA (2018), entre el 2014 y 2016, muestran que la tasa de mortalidad ha sido inestable de un año a otro si se considera la variabilidad entre las entidades federativas, las cuales se han desviado respecto a la media nacional entre 4 a 5 defunciones, más o menos, por cada 100000 habitantes $\left(\mathrm{DE}_{2014}=4.49 ; \mathrm{DE}_{2015}=4.4 ; \mathrm{DE}_{2016}=\right.$ 5.48). En cuanto a los variaciones en cada entidad, algunas son estables en su tasa de mortalidad entre 2015 y 2016, como son los casos de Yucatán ( $\mathrm{DE}=0.1)$, Michoacán $(\mathrm{DE}=0.21)$, Hidalgo $(\mathrm{DE}=0.21)$, y Querétaro
$(\mathrm{DE}=0.3)$; por otra parte hay entidades que han experimentado un mayor cambio en su tasa de mortalidad en el período referido, como Coahuila $(\mathrm{DE}=3.1)$, Nayarit $(\mathrm{DE}=2.99)$, y Zacatecas $(\mathrm{DE}=2.72)$. Lo anterior no implica que las entidades federativas de menor o mayor variación en el período de 2014 a 2016 tengan las menores o mayores tasas de mortalidad, solo ilustra que el panorama mexicano es muy heterogéneo respecto a los accidentes de tránsito y sus consecuencias, así podemos observar que entidades como Veracruz, Ciudad de México, y el Estado de México no rebasan las 10 defunciones por cada 100,000 habitantes, mientras otros exceden las 20 defunciones, como Nayarit, Durango, Sinaloa, Tabasco y Zacatecas.

Ante los compromisos enunciados por México desde el 2011 y las cifras anteriormente presentadas sobre accidentalidad y 
Carro, E.

mortalidad, surgen preguntas como las siguientes: ¿por qué no se han alcanzado las metas enunciadas relativas a la mortalidad?, ¿qué aspectos de las estrategias emprendidas para reducir la cantidad de accidentes y las tasas de mortalidad han funcionado y cuáles no?, ¿por qué la reducción en los accidentes de tránsito, con el tiempo, parece estar asociada a un incremento de los decesos?

Sí bien las respuestas pueden ser diversas, una parece plausible y sustentada en los planteamientos de la OMS (2011) sobre un sistema seguro, en el cual no solo son responsables de los accidentes los usuarios de las vías de tránsito, sino también las autoridades encargadas de vigilar el cumplimiento de los reglamentos, y los diseñadores de vías y vehículos, en suma, el porqué de los accidentes son los seres humanos que emiten respuestas, posicionamientos, creencias sobre lo que es seguro o no, prescindible o no en seguridad vial, respuestas que en conjunto podríamos resumirlas en un término: comportamiento vial.

Considerando lo anterior, una de las tareas que se aprecia fundamental para coadyuvar a mejorar la situación actual sobre seguridad vial en el país, es la de incrementar las iniciativas de investigación e intervención para el estudio y modificación del comportamiento vial. Tarea congruente con los pilares dos, tres y cuatro del plan mundial para la seguridad vial, relativos a Vias de tránsito y movilidad más seguras, Vehículos más seguros, y Usuarios de vías de tránsito más seguros, respectivamente (OMS, 2011). El fomento y desarrollo de estas iniciativas de investigación e intervención en otros contextos nacionales ha mostrado buenos resultados (Soler y Tortosa, 1985; Tortosa y Montoro,

Tabla 4

Áreas de investigación e intervención para una Psicología del Tránsito en México.

\begin{tabular}{|c|c|c|}
\hline Áreas o dimensiones & Pilares de la OMS & Líneas específicas de trabajo \\
\hline $\begin{array}{l}\text { Comportamiento de los usua- } \\
\text { rios }\end{array}$ & Pilar 3 y 4 & $\begin{array}{l}\text { - Determinantes de la emisión de conductas de riesgo por } \\
\text { parte de los conductores de vehículos: velocidad de con- } \\
\text { ducción, uso de celular, dispositivos de seguridad, y } \\
\text { otros. } \\
\text { - Movilidad del peatón y conductas de riesgo. } \\
\text { - Factores asociados al comportamiento preventivo y } \\
\text { mantenimiento del automóvil. } \\
\text { - Modificación de conductas de riesgo en los usuarios. } \\
\text { - Capacitación de usuarios. }\end{array}$ \\
\hline $\begin{array}{l}\text { Comportamiento de las auto- } \\
\text { ridades }\end{array}$ & Pilar 2 & $\begin{array}{l}\text { - Determinantes en la aplicación de las normas o regla- } \\
\text { mentos de tránsito a los usuarios. } \\
\text { - Evaluación de marcos regulatorios y sus efectos en el } \\
\text { comportamiento de riesgo. } \\
\text { - Factores asociados a la planeación de estrategias pre- } \\
\text { ventivas y de mitigación. } \\
\text { - Capacitación de autoridades de tránsito. }\end{array}$ \\
\hline $\begin{array}{l}\text { Comportamiento de planifica- } \\
\text { dores }\end{array}$ & Pilar 2 & $\begin{array}{l}\text { - Definidores en la planificación de las vías de tránsito. } \\
\text { - Valor y concepciones sobre los usuarios en la planifica- } \\
\text { ción de las vialidades. } \\
\text { - Diseño de vialidades eficiente en base a principios psi- } \\
\text { cológicos y psicosociales. }\end{array}$ \\
\hline $\begin{array}{l}\text { Evaluación de las intervencio- } \\
\text { nes }\end{array}$ & Pilar 2,3 y 4 & $\begin{array}{l}\text { - Evaluación de marcos regulatorios y sus efectos en el } \\
\text { comportamiento de riesgo de los usuarios de las vías. } \\
\text { - Características de las vías y sus efectos en el comporta- } \\
\text { miento de los usuarios. } \\
\text { - Evaluación de las estrategias de prevención y mitiga- } \\
\text { ción de accidentes de tránsito y sus consecuencias. }\end{array}$ \\
\hline
\end{tabular}


2002).

Este desarrollo de mayor investigación e intervención en el área del comportamiento vial solo se puede lograr sí existe, de igual forma, un mayor involucramiento de las disciplinas científicas encargadas del estudio del comportamiento humano, entre ellas la psicología del tránsito, la cual en México apenas empieza a despertar intereses (Dorantes, Cerda, Tortosa y Ferrero, 2015; Mirón y Laborín, 2016).

De tal manera, urge la promoción de la psicología del tránsito en los sectores formativos, de investigación y prevención, psicología que de acuerdo al panorama mexicano deberá inicialmente estar comprometida en el diseño y desarrollo de investigación enfocada al conocimiento de los factores intervinientes en los accidentes de tránsito y tasas de mortalidad actuales, y posteriormente en el campo de la prevención y mejoramiento de las vías de tránsito. A manera de colofón se propone un conjunto de líneas de trabajo integradas a cuatro áreas o dimensiones de estudio, que se asocian a los pilares dos, tres, y cuatro de la OMS (2011), y que pueden resultar prometedoras para una psicología del tránsito en México. (Ver Tabla 4).

\section{Agradecimientos}

Artículo realizado gracias al apoyo otorgado por la Dirección General de Educación Superior Universitaria, PRODEP México, Apoyo de Fomento a la Generación y Aplicación Innovadora del Conocimiento, mediante oficio 511-6/17-8212 al proyecto PERCEPCIÓN DE RIESGO Y CONDUCTAS DE RIESGO ASOCIADAS AL CONDUCIR UN AUTOMÓVIL EN ZONAS URBANAS DEL SUR DE TAMAULIPAS Y CIUDAD DE MÉXI$\mathrm{CO}$.

\footnotetext{
Referencias

Declaración de México para la Seguridad Vial: la ruta para salvar vidas en Iberoamérica y el Caribe (13 de mayo de 2011). EISEVI ${ }^{2}$. 2do encuentro Iberoaméricano y del caribe sobre seguridad vial. Recuperado el 23 de Octubre de 2018, de https://www.paho.org/mex/ index.php?
}

option=com_docman\&view=download\&category_slug= estrategia\&alias $=741$-declaracioneisevi2\&Itemid $=493$

Dorantes, G., Cerda, E. A., Tortosa, F., y Ferrero, J. (2015). Accidentalidad de automóviles de uso particular en México: influencia del estrés y la agresividad. PSIENCIA. Revista Latinoamericana de Ciencia Psicológica, 7(3), 418-427. doi: 10.5872/psiencia/7.3.122

Estrategia Nacional de Seguridad Vial 2011-2020. Diario Oficial de la Federación. (6 de junio de 2011) Recuperado el 30 de Octubre de 2018, de http://dof.gob.mx/ nota_detalle.php? codigo $=5193284 \&$ fecha $=06 / 06 / 2011$

Mirón, C.A y Laborín, J.F. (2016). Características psicométricas de escala de heurísticos de disponibilidad en transgresión de alto en jóvenes conductores. Revista de Psicología y Ciencias del Comportamiento de la Unidad Académica de Ciencias Jurídicas y Sociales, 7 (2), 52-67. doi:10.29365/rpcc.20161125-52

Organización Mundial de la Salud [OMS ] (2011). Plan Mundial para el Decenio de Acción para la Seguridad Vial 2011-2020. Recuperado el 24 de Abril de 2011, de http://www.who.int/roadsafety/decade_of_action/plan/ plan_spanish.pdf?ua $=1$

Secretariado Técnico del Consejo Nacional para la Prevención de Accidentes [STCONAPRA] y Secretaría de Salud 2013). Perfil Nacional de Seguridad Vial, México 2013. Recuperado el 6 de Octubre de 2018, de http:// www.conapra.salud.gob.mx/Interior/Documentos/ Observatorio/

Perfiles/0_Perfil_Nacional_Accidentes_Transito.pdf

Secretariado Técnico del Consejo Nacional para la Prevención de Accidentes [STCONAPRA] y Secretaría de Salud (2016). Informe sobre la situación de la seguridad vial, México 2015. Recuperado el 15 de Marzo de 2017, de http://www.saludzac.gob.mx/home/images/ programas/Informe2015.pdf

Secretariado Técnico del Consejo Nacional para la Prevención de Accidentes [STCONAPRA] y Secretaría de Salud (2018). Informe sobre la situación de la seguridad vial, México 2017. STCONAPRA-SS: Ciudad de México. Recuperado el 9 de Septiembre de 2018, de https://drive.google.com/file/ d/1IcEIsytf4rET_pZVdQWA-bXbAfwcenFA/view

Soler. J. y Tortosa, F. (1985). Psicología y seguridad vial: autores, temas y fuentes. Papeles del Psicólogo. 21(4) 3237 Recuperado el 7 de Marzo de 2012, de http:// www.papelesdelpsicologo.es/vernumero.asp? $\mathrm{id}=233$

Tortosa, F. y Montoro, L. (2002). La Psicología aplicada a la selección de conductores. Cien años salvando vidas. Psicothema. 14(4), 714-725. 